\title{
Transposition
}

Musique et Sciences Sociales

Hors-série 1 | 2018

Musique, histoire, sociétés

\section{Le séminaire de Tran Van Khê à l'EHESS}

\section{François Picard}

\section{OpenEdition}

Journals

Édition électronique

URL : http://journals.openedition.org/transposition/1721

DOI : 10.4000/transposition. 1721

ISSN : 2110-6134

\section{Éditeur}

CRAL - Centre de recherche sur les arts et le langage

\section{Référence électronique}

François Picard, «Le séminaire de Tran Van Khê à l'EHESS », Transposition [En ligne], Hors-série 1 | 2018, mis en ligne le 30 janvier 2018, consulté le 11 février 2020. URL : http:// journals.openedition.org/transposition/1721 ; DOI : 10.4000/transposition.1721

Ce document a été généré automatiquement le 11 février 2020.

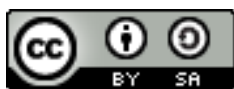

La revue Transposition est mise à disposition selon les termes de la Licence Creative Commons Attribution - Partage dans les Mêmes Conditions 4.0 International. 


\section{Le séminaire de Tran Van Khê à l'EHESS}

\section{François Picard}

1 Au milieu des années 1980, un séminaire de musicologie s'est tenu à l'École des hautes études en sciences sociales. De musicologie, mais aussi plutôt de sciences sociales de la musique, et encore portant sur une culture vivante géographiquement située, autrement dit de ce que l'on appellerait aujourd'hui de l'ethnomusicologie: le séminaire sur "Les chants alternés en Asie du Sud Est» de Tran Van Khê, alors directeur de recherches au CNRS et membre de l'équipe CNRS-Langues'O Centre de Documentation et de Recherche sur l'Asie du Sud-Est et du Monde Insulindien (CeDRASEMI).

2 Une des traces de cette histoire figure dans une des nombreuses bibliographies $d u$ maître disséminées sur la Toile ${ }^{1}$, une autre dans un document de l'Association française pour la recherche sur l'Asie du Sud-Est (AFRASE) daté de février 1985. D'autres traces ont marqué ma formation à l'ethnomusicologie et mon parcours, de musicien à musicologue, de la scène à l'estrade ${ }^{2}$. Il a paru utile, on espère nécessaire, de retracer un moment peu présent dans les annales, et même dans les mémoires, où se constitua une alliance entre étude de la musique et sciences sociales, hors des musées, des conservatoires et même des programmes universitaires.

\section{Ethnomusicologie et études des musiques orientales : le paysage intellectuel en 1985}

3 En 1985, le séminaire de Bernard Lortat-Jacob au Musée de l'Homme réunit Gilbert Rouget et Simha Arom, Hugo Zemp et André-Marie Despringre, avec Pribislav Pitoëff et Tran Quang Haï, Monique Brandilly et Mireille Helffer (tous présents par exemple le 21 octobre 1985 sauf cette dernière). La Société française d'ethnomusicologie (SFE) vient d'être créée en 1983, sous l'impulsion de Maurice Fleuret, directeur de la Musique de Jack Lang, ministre de la Culture. 
4 Le Musée de l'Homme et la SFE, avec le musée des Arts et Traditions Populaires qui reste bien en dehors, semblent rassembler tout ce qui se fait en enseignement et recherche. On notera le cours de Claude Laloum à Paris 8 Vincennes, désormais à SaintDenis, et le recrutement du premier enseignant-chercheur titulaire, Michel de Lannoy, à l'université de Tours. Cependant, Akira Tamba, Jean-Claude Chabrier et Tran Van Khê, tous trois du $\mathrm{CNRS}^{3}$, ne fréquentent guère les autres ethnomusicologues, termes que d'ailleurs chacun d'eux récuse ou évite; tous trois étudient en effet des musiques de l'Asie (respectivement Japon, Proche-Orient et Viêt Nam), qui ont des histoires, des théories, et ne sauraient selon eux être limitées à un présent collectif et un caractère oral. Tran est de plus en contact avec d'autres chercheurs d'envergure internationale (le Japonais Kishibe Shigeo, l'Indien Narayan Menon, le Philippin José Maceda, le Taiwanais Hsü Tsang-Houei) qui se rencontrent au sein du Conseil international de la musique (fondé en 1949 auprès de l'UNESCO) comme du Conseil international des musiques traditionnelles, dont il est en 1982 vice-président. Mais malgré son attachement à la Sorbonne qui l'a accueilli, formé et diplômé, Tran ne parvient pas à y diriger des thèses en personne, ni à y développer un enseignement de musique et musicologie de l'Asie du Sud-Est et Orientale ${ }^{4}$. Pour s'y employer, il va fonder une association, le CEMO, et créer un séminaire à l'EHESS.

\section{Le CEMO}

Musique, musicologie, sciences sociales, expérience de terrain, se former à l'ethnomusicologie à une époque ou dans des lieux où un cursus complet n'existe pas nécessite un parcours marqué par des fausses pistes, des rencontres, des indications, des relais, des repos, des retards. Je suis venu à ce domaine par l'étude d'une flûte traversière à six trous de jeu (flûte d'Allemagne copie de l'époque Renaissance) qui m'a conduit en novembre 1983 au dizi 笛子 chinois, dont ma sœur Nathalie m'avait rapporté un exemplaire de Chine et que Cheng Shui-cheng enseignait au Centre d'études de musique orientale (CEMO), une association. Les cours avaient lieu à la faculté des sciences, autrement dit à Jussieu, ou encore "La halle aux vins », accueillis au département de mécanique (physique) par le Laboratoire d'acoustique musicale (LAM) d'Émile Leipp et Michèle Castellengo, Tour 66, $5^{\mathrm{e}}$ étage, les lundis de $18 \mathrm{~h}$ à $20 \mathrm{~h}$, Castellengo délivrait son fameux cours d'acoustique musicale hors cursus, dont la première séance eut lieu le 7 novembre 1983.

$6 \mathrm{Au}$ CEMO je rencontre Alain Desjacques, ethnomusicologue et comme moi flûtiste, spécialiste de Mongolie, devenu plus tard maître de conférences à Lille, et quelques chanteurs chinois : Shi Kelong (时可龍), Shi Peipu (时佩橏), qui me demande bientôt de l'accompagner en concert, puis d'écrire ses mémoires. Mais ce dernier sera bientôt pris dans une tourmente politico-judiciaire, un roman d'amour et d'espionnage dont on peut espérer que Roger Darrobers, sinologue, fera un jour le récit ${ }^{5}$.

7 Fondé en 1959, le CEMO, pompeusement placé sous l'égide de l'Institut de musicologie de la Sorbonne, est une école de musique associative. Elle compte parmi ses enseignants le gratin des musiques orientales: d'abord Tran Van Khê lui-même à la cithare vietnamienne, puis à partir de 1961 Djamchid Chemirani au tambour persan zarb, auquel succède en 1978 Brunot Caillat qu'il a formé 6 , rejoints par Dariush Talai, aux luths persans tar et setar de 1979 à 1989, à partir de 1977 Talip Özkan aux luths turcs, rejoints par d'autres ensuite. 
En 1987-1988, le centre compte dans ses enseignants Tran Van Khê, Tran Thi, Thuy Ngoc (cithare đàn tranh, Viêt Nam); Cheng Shui Cheng (luth pipa, vièle nanhu, flûte droite xiao), Li Ying (cithare zheng, Chine); Narendra Bataju et Patrick Moutal (sitar), Krishna Govinda et Brunot Caillat (tabla), Jean-Paul Auboux (flûte carnatique, Inde); Hossein Omoumi (flûte ney, répertoire de chants), Dariush Talai et Jean During (luths tar et setar), Mahmoud Tabrizi-Zadeh (vièle kemantche), Massoud Omidi (cithare santour) ; Brunot Caillat, Madjid Khalaj, Zia Mirabdolbaqi (zarb ou tombak, Iran); Aram Kerovpian (cithare sur table kanun, trad. Arménienne); Marc Loopuyt (luth oud, tradition arabe et turque), Talip Özkan (luths tambur et saz) Kudsi Erguner (ney, tradition turque), Kamal Morkos (chant arabo-andalou, mouachahaat).

Un programme de concert du 16 janvier 1986 à l'amphithéâtre Richelieu de la Sorbonne réunit ainsi musiques du Viêt Nam avec Tran Van Khê : chant, luth, vièle, Thuy Ngoc : cithare; de Chine avec Cheng Shui Cheng : luth pipa, Shi Pei Pu : chant, Li Ying : cithare zheng; de l'Iran avec Hossein Omoumi : flûte ney, chant, Bruno Caillat : zarb; d'Inde du Sud avec Jean-Paul Auboux : flûte, Bruno Caillat : tambour mridangam et luth tanpura.

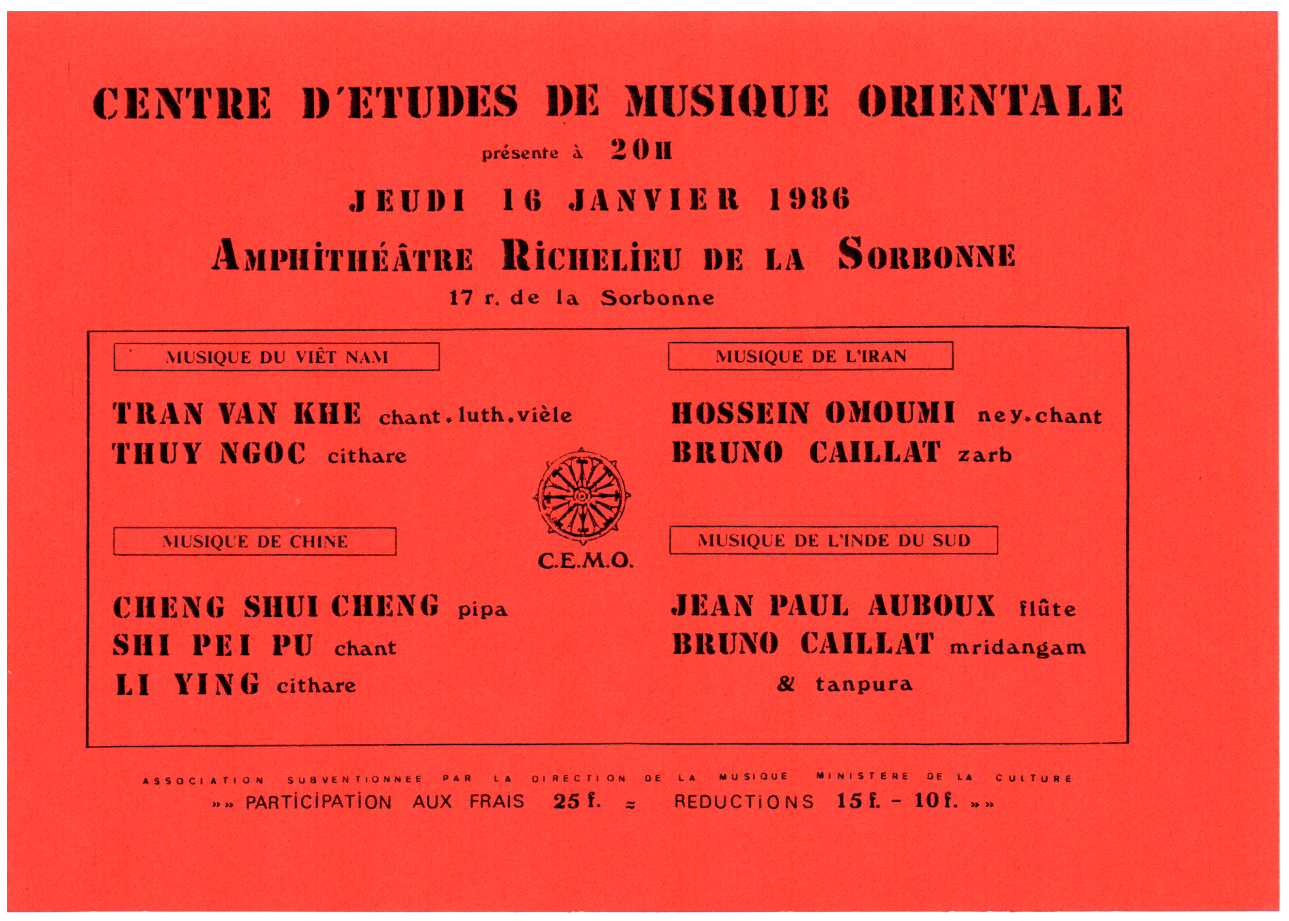

Le CEMO fournissait une respectabilité à travers la Sorbonne à ses enseignants, qui se prévalaient volontiers du titre visiblement prestigieux de "professeur à la Sorbonne " (aucun ne l'était, même Tran), la présidence ou la direction en était également signe de distinction, puisque non seulement Tran ${ }^{7}$ mais aussi Alain Daniélou ${ }^{8}$ se prévalent de l'avoir dirigé, présidé, voire fondé... alors qu'il faudrait avant tout ne serait-ce que citer ici le nom de Denise Jourdan-Hemmerdinger, musicologue spécialiste de la Grèce antique, chercheuse au CNRS, qui en fut la secrétaire générale9. Inversement, un professeur des universités en titre et en poste à la Sorbonne pouvait se prévaloir du CEMO pour asseoir sa réputation d'expert des musiques d'Orient: Manfred Kelkel ${ }^{10}$, tout comme Lloyd Miller, musicien et musicologue de Salt Lake City, pionnier du jazz oriental, qui le fréquenta dans les années 1960.

11 Mais Tran Van Khê désirait donner un aspect universitaire, intellectuel, si ce n'est de recherche, à l'enseignement des pratiques musicales orientales. C'est ainsi qu'il rend 
obligatoire pour ceux qui suivent les cours de musiques vietnamienne ou chinoise les cours "Chine Musique populaire du peuple Han ", "Musique populaire du Vietnam " ou encore le cours théorique "Chine. Histoire de la musique", "Chine. Théorie musicale » de Cheng les samedis de $10 \mathrm{~h}$ à $12 \mathrm{~h}$. Il accueille aussi le 5 novembre $1984 \mathrm{en}$ Sorbonne une conférence du professeur Hsu Tsang-Houei 許常惠, de Taiwan, compositeur, musicologue et ethnomusicologue.

Et puis un jour d'octobre 1984, voyant que je suivais bien les cours d'histoire et de théorie, que je prenais des notes et étais assidu, Tran Van Khê m'interroge - il apprend sans doute alors que je suis titulaire d'un DEA (Études techniques et esthétiques du théâtre, Paris 8 Vincennes, 1977) - et m'invite à suivre son séminaire à l'EHESS. C'est ainsi que je découvre l'immeuble du 44 rue de la Tour, à Paris $\mathrm{XVI}^{\mathrm{e}}$ (une excellente pâtisserie y proposait des palmiers de première importance), les vendredis de $10 \mathrm{~h}$ à $12 \mathrm{~h}$. C'est dans ce bâtiment qu'étaient logés l'Association française pour la recherche sur l'Asie du Sud-Est et le Centre de documentation et de recherche sur l'Asie du SudEst et le monde insulindien.

13 À l'EHESS, il y a Françoise Escal (née Rué, 1935-2015, maître assistant à Paris X en 1982, alors membre du CESTA Centre d'études transdisciplinaires d'Edgar Morin et Gérard Genette), directrice d'études, non loin François Lesure à l'École pratique des hautes études (EPHE). François-Bernard Mâche est encore à Strasbourg. Quand, inspiré par Tran Van Khê et désireux de rentrer en musicologie, j'écris un projet de thèse, je le soumets, entre février et juillet 1985, à Michèle Castellengo (Paris 6), Edgar Morin (EHESS), Tran Van Khê (Paris IV, mais il ne dirige pas directement), François Lesure (EPHE), Iannis Xenakis (Paris 1), et le communique à Michel Philippot (CNSM), Alain Bancquart (CNSM), Jean-Baptiste Barrière (IRCAM). J'ai beau suivre le séminaire d'ethnomusicologie du Musée de l'Homme, il est exclu que je puisse y faire ma thèse.

\section{Le Centre de documentation et de recherche sur l'Asie du Sud-Est et le monde insulindien (CeDRASEMI)}

Puisque l'enseignement sur les musiques du Viet Nam et de la Chine s'inscrivent ainsi dans le cadre de sciences sociales, un peu de contexte, qui manquait alors totalement à un simple apprenti aficionado des musiques orientales, doit être connu. Le CeDRASEMI fondé en 1962 comme Recherche Coopérative sur Programme du CNRS par Georges Condominas (1921-2011), parangon de l'ethnologue sérieux (terrain lointain, populations forestières, langues étranges), a été délocalisé à Sophia-Antipolis Valbonne en 1981, avant d'être démantelé par Maurice Godelier (1934-) en 1984. Le séminaire du mardi matin de «Condo » s'est déroulé longtemps rue de la Tour.

Menacés, puis acculés, les chercheurs réagissent en sortant du bois vers la place publique : ils fondent le 23 juin 1984 l'Association française pour la recherche sur l'Asie du Sud-Est. Les membres du CeDRASEMI proposent ${ }^{11}$ à l'EHESS « en plus de séminaires principaux de Condominas, Daniel de Coppet et Denys Lombard», les trois directeurs d'étude en titre, des "conférences complémentaires", parmi lesquelles "Les chants alternés en Asie du Sud Est » par Tran Van Khê, CNRS. 
CONFERENCES COMFLENTAIRES (harS DEA)

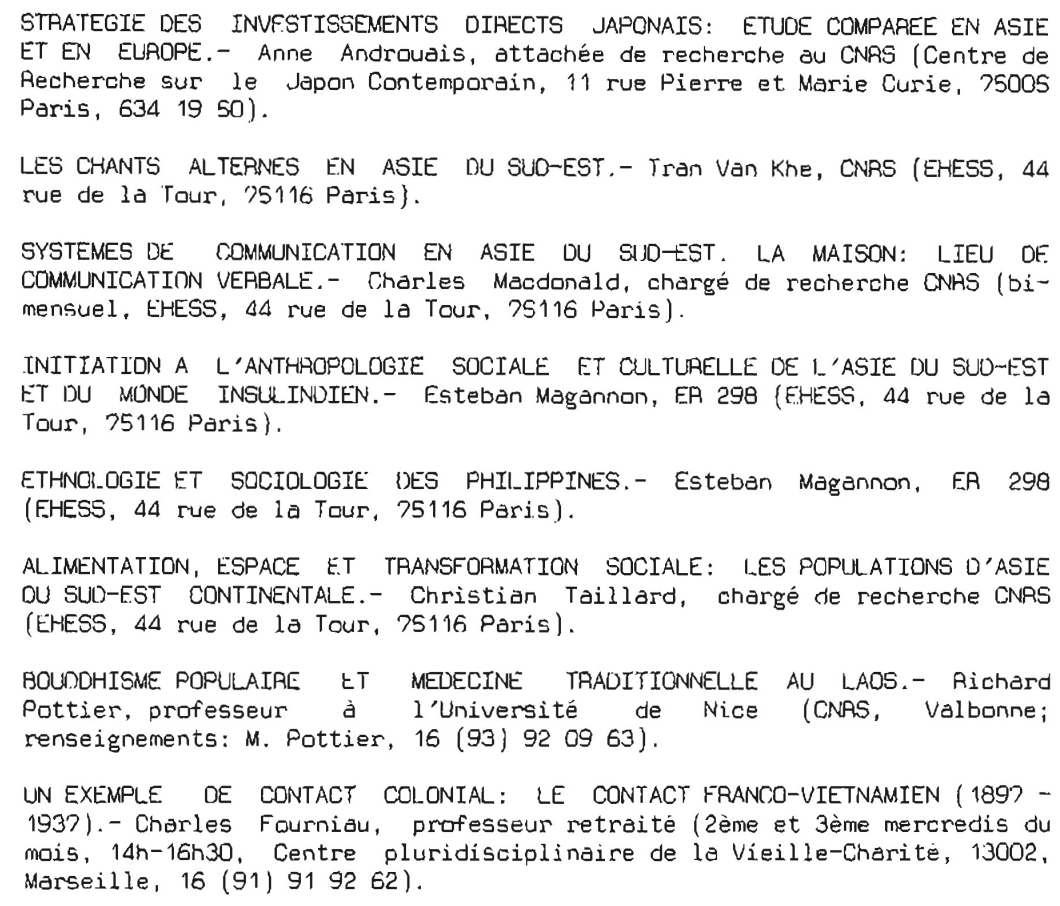

\section{Les contenus}

16 Nous allons successivement détailler l'enseignement donné au CEMO et à l'EHESS, dont on verra ainsi la complémentarité et la similarité. Les listes ci-dessous sont tirées de mon carnet de notes et de mes feuilles de cours manuscrites.

\section{Cheng Shui-Cheng, Tran Van Khê : cours théorique 1984-1985 «Chine»}

Les samedis $9 \mathrm{~h}-11 \mathrm{~h} 30$, Jussieu, Tour $66,5^{\mathrm{e}}$ étage

La notation chinoise

Théorie musicale

- point de vue philosophique

- point de vue physique

Survol de l'histoire de la musique chinoise, des genres

Classification des instruments

Musique populaire, chants de travail

7 janvier 1984

25 janvier 1984 « Chants alternés »; les échelles par le cycle des quintes; le mode

11 février 1984

21 avril 1984 «Musique fonctionnelle »; même fonction pour plusieurs usages, un usage pour plusieurs fonctions ; exemple du chant de repiquage

28 avril 1984 (On chante les chants familiaux en cas de désaccord; à la guerre, pour démoraliser l'adversaire, les soldats chantaient les airs du pays de leur adversaire) 19 mai 1984 « Musique populaire »;

5 novembre 1984 Prof. Hsü « Musique de Taiwan » 
5 janvier 1985 « La notation » : nom des notes, doigtés

19 janvier 1985 Tran Van Khê, "Théorie de la musique chinoise»: mozisme, confucianisme, taoïsme

26 janvier 1985 Tran Van Khê, « Théorie de la musique chinoise » : le président Mao

2 février 1985 Tran Van Khê, "Théorie de la musique chinoise » : les cinq catégories :

des peuples minoritaires; traditionnelles; savantes de cour; savantes de théâtre; religieuses

9 février 1985 Tran Van Khê, «Théorie de la musique chinoise » : les cinq catégories

2 mars 1985 Tran Van Khê, "Théâtre traditionnel de Beijing "; musique contemporaine 20 avril 1985 « Chants de travail des peuples minoritaires de Taiwan »

9 novembre 1985

16 novembre 1985

23 novembre 1985 Tran Van Khê, «Histoire de la musique chinoise » cithares santur, yangqin, yanggŭm, đàn tam thập lục

30 novembre 1985

7 décembre 1985 « Tétratoniques et pentatoniques »

14 décembre 1985 « Les notations musicales " gongche, cordes du koto, air Chunguang wu 春光舞 (Danse du rayon printanier) en notation gongche et jianpu; Rousseau, " dissertation sur la musique moderne »

11 janvier 1986 Tran Van Khê, « Histoire de la musique chinoise » cithare guqin, thèse de Yoko Mitani, Yip Ming Mei, David Mingyue Liang, Van Gulik.

\section{Tran Van Khê, séminaire}

C'est donc dans le contexte de survie du centre d'études de l'Asie du Sud-Est que Tran, comme les autres chercheurs, offrent ces séminaires. En bénéficient: François Picard, musicien et technicien de théâtre, intermittent du spectacle; Shi Kelong, chanteur, professeur au Conservatoire de Chine (Pékin), en formation à l'Opéra Comique auprès de Michel Sénéchal, directeur artistique de l'ensemble Fleuve Jaune. Et durant un an, Nicole Revel Macdonald, chercheuse CNRS sur Palawan, que le maitre tente d'initier aux rudiments de la musicologie du pentatonisme. Soit donc zéro étudiants inscrits.

Cette gratuité de l'enseignement est une merveille de l'institution de l'enseignement supérieur français, symbolisé par le Collège de France : au sommet de la pyramide d'excellence, cette institution enjoint aux chercheurs d'enseigner et leur interdit d'avoir des étudiants auxquels ils conféreraient un diplôme - cruauté tempérée par l'institution à l'EPHE et à l'EHESS du directeur d'études cumulant. Outre le séminaire de Tran Van Khê, j'ai ainsi bénéficié à l'EPHE V d'une extraordinaire série de cours sur le bouddhisme chinois par Hubert Durt, chercheur permanent à l'École française d'Extrême Orient (EFEO) et d'un merveilleux cours de Jean-Noël Robert sur le bouddhisme sino-japonais à l'EPHE : là aussi nous étions zéro étudiants inscrits. Robert sensei est désormais professeur au Collège de France et ses deux étudiants d'alors sont le premier professeur à Paris-Sorbonne et le second, Pierre Marsone, directeur d'études à l'EPHE IV. C'est cette ouverture, cette gratuité, cette offre sans limite qui a formé une génération de chercheurs enseignants pluridisciplinaires. 


\section{Le séminaire, intitulés et contenus}

À l'intérieur de son séminaire au titre bien délimité, comme c'est l'usage à l'EPHE et à l'EHESS, Tran distille des concepts généraux. Il distingue rigoureusement fonction et usage, met en valeur qu'une même fonction peut correspondre à plusieurs usages, et un usage à plusieurs fonctions. Il détaille les procédés de textures : contrepoint, mélodie à bourdon, hétérophonie, et les procédés formels: canon, tuilage, responsorial, antiphonal, strophique, à ritournelle. Il inscrit son enseignement à l'EHESS, dans le cadre de recherches interdisciplinaires sur le riz et l'eau qui donneront lieu à une publication collective $^{12}$; il donne des fondements théoriques puisés chez Henri Davenson, André Schaeffner, Julien Tiersot, distinguant Rezeptions theorie et théorie romantique.

21 Les vendredis de $10 \mathrm{~h}$ à $12 \mathrm{~h}$

2 octobre 1984 Tran Van Khê, «Les chants alternés par garçons et filles en Asie » 11 janvier 1985 Tran Van Khê, «Les chants alternés par garçons et filles en Asie » 25 janvier 1985 Tran Van Khê, «Les chants alternés par garçons et filles en Asie » 26 avril 1985 Tran Van Khê, « Les chants alternés par garçons et filles en Asie » 10 mai 1985 Tran Van Khê, « Les chants alternés par garçons et filles en Asie » $1^{\text {er }}$ février 1985 Nicole Revel-MacDonald, «Les chants alternés aux Philippines et aux Insulindes 》

15 février 1985 Tran Van Khê, « Chants alternés du Viet Nam » $1^{\text {er }}$ février 1985 Nicole Revel-MacDonald, « Kulilal » chants alternés des Kalinga

22 mars 1985 Tran Van Khê, «Les chants alternés en Asie du Sud-Est »

26 avril 1985 Cheng Shui-Cheng, «Les chants alternés de Chine méridionale et de Formose »

3 mai 1985 Cheng Shui-Cheng, «Liu Sanjie », film tiré d'une opérette

10 mai 1985 Tran Van Khê, «Les chants alternés en Asie du Sud-Est » qui ? quoi ? où ? quand? comment?

$1^{\text {ers }}$ et $3^{\text {es }}$ jeudis de chaque mois

7 novembre 1985 Tran Van Khê, « Chants alternés d'Asie du Sud-Est »

\section{Quelques enseignements}

Il y a un monde entre la compétence musicale et musicologique de Tran et celle de certains de ceux qui suivaient son séminaire. Comme il y a un monde entre le profond investissement en temps et en sentiments sur le terrain, avec les gens, de Nicole Revel et le recueil de données sur le terrain pratiqué par Tran lors de présentations organisées ou l'analyse de documents de troisième main (un film romancé) par Cheng.

Tran nous enseignait qu'il fallait documenter nos entretiens et enregistrements, et apporter la preuve en fournissant et publiant les photos de l'ethnologue avec les informateurs. J'avais bien quelque doute, et j'ai bien ri quand, sur le Bund, la berge sur la rivière Huangpu à Shanghai, des millions de Chinois venus de tout le pays se faisaient prendre en photo, sans doute pour montrer que c'était bien eux et qu'ils y étaient bien.

Ce mélange extraordinairement fin entre le savant européen et le bateleur asiatique faisait toute la saveur des interventions de $\operatorname{Tran}^{13}$. Quel que soit le lieu : concert, soirée privée entre lettrés, cours au CEMO ou en Sorbonne, communication dans une 
conférence internationale, séminaire à l'EHESS, il ne changeait jamais de style, usait des mêmes trucs et des mêmes ficelles, se mettait le public dans la poche, impressionnait par sa capacité à mobiliser les langues, les savoirs intellectuels, la précision de la formulation et la vivacité à faire l'aller-retour parler-chanter. Son séminaire, toujours courtois, serein, amical, était l'occasion de rencontres extraordinaires. J'ai pu y rencontre ainsi en invités spéciaux Mireille Helffer, directrice de recherche au CNRS (musiques du bouddhisme tibétain), Georges Condominas (ethnologue, Viêt Nam), José Maceda (compositeur, musicologue, ethnomusicologue, Philippines) et sans doute Claudine Salmon (ethnologue, Indonésie).

Il m'a légué des principes sûrs en termes d'analyse des musiques modales et pentatoniques, l'importance de l'engagement artistique pour réaliser des travaux sur l'art, la distinction entre fonction et usage : par exemple, un chant entonné lors d'un rituel n'a pas pour autant fonction rituelle, une absolue absence de racisme ou de rancœur post-coloniale. On trouvera ci-dessous la liste de quelques documents distribués et conservés, et l'édition d'un document particulier : le chant alterné « Mời giầu $»^{14}$.

\section{Annexe : Tran Van Khê, documents distribués}

TRAN, Van Khê, Bibliographie sommaire sur les chants alternés en Asie du Sud-Est, dactylogramme, 101 item, $14 \mathrm{p}$.

TRAN, Van Khê, «La musique populaire au Vietnam, Ses attaches avec la musique de tradition savante ou la musique rituelle", International Folk Music Journal 18 (1966), p. 2-14.

Chant d'amour 山歌愛, transcription et traduction du texte, notation musicale.

Liu Sanniang劉三姐, transcription et traduction du texte, notation musicale. Film de 1960, réalisation Su Li苏里, compositeur Lei Zhenbang 雷振邦, avec Huang Xangqiu黄 婉秋, Li Shilong 刘世龙, Xia Zongxue 夏宗学 Moi giau (inviter le visiteur à prendre du bétel), notation musicale.

\section{Chant d'amour 山歌愛, transcription et traduction du texte, notation musicale.}


以賺錢還債，但遇到土匪，失去音訊，秀秀等了三天三夜，以為他給殺死了，另一 方面,

曾老虎又迫他們還錢，不得已秀秀只好下嫁，成親時大龍竟然生還，去追花轎，和 曾老虎

打了一架, 雖然輸了, 但曾老虎看他們兩情相悅, 就默默退出, 成全他們.

少年比唱

少年 : 哎! 山歌一唱月放光噯, 山歌一唱花開放噯, 花月也都知情意噯, 姑娘為何 不比唱噯?

秀秀: 哎! 花兒比香我比唱嗳, 鳥鴉怎能比鳳凰噯, 你能唱得月放光噯, 我能唱得 龍出江噯.

少年 : 花兒開了花飄香噯，女兒大了找情郎噯，姑娘年紀十八九噯，問妳心裡把誰 想噯?

秀秀 : 鳥鴉開口叫呱呱噯, 狗嘴怎能長象牙噯, 你要問誰想出嫁噯, 問你姐妹想那 家噯.

少年 : 十八十九大姑娘噯, 送妳白布妳嫌髏噯, 再過三年花落了嗳, 看妳兩眼淚汪 汪噯.

秀秀: 野狗沒窩到處逝噯, 姑娘門前叫汪汪噯, 罵不走來趕不跑嗳, 棍子味道你嚐 嚐噯.

www.amychan.info/ying/lingboh/yellow/hill2.htm

自50年代末，香港流行著一片黃梅調熱潮，除了開拍電影外，黃梅調唱片也是非常流 行，同期來自大陸劉三姐的熱潮，也出現了另一種“山歌”歌唱片，1964年香港便有山歌 戀上演。

http://blog.roodo.com/muzikland/archives/1649944.html

\section{Moi giau (inviter le visiteur à prendre du bétel), notation musicale.}

Chanté par Hai Cai et Nam Dan le 14-03-76 à Bắc Ninh, enregistrement et transcription par tran Van Khê, même chant que «Dạy hát Quan Họ» http:// www.quanhomuoinho.com/video/day-hat-quan-ho-moi-giau/ à 7’30

Thì tay ớ em ớ nâng í cái cơi có đựng í a à a giầu.

Mắt í em nhìn, nhìn em liếc ơ liếc em trông i cái cơi có đựng í a à a giầu.

Giầu têm a ới à a hư hừ là, cánh i phượng cũng rằng là dâng lên, dâng ớ ơ lên là lên em mời, ì í a à à người, ứ hự ư hự người ơi.

Ai ơi nay có thấu, người ơi nay có nhớ ơ chăng i, chăng là đến chúng em chăng i.

Ai ơi nay có thấu, người ơi nay có nhớ ơ chăng i, chăng là đến chúng em chăng i. ứ hư ư hự hời hư

http://quanhobacninh.vn/video/hoc-hat-quan-ho-moi-nuoc-moi-trau/ 


\section{BIBLIOGRAPHIE}

Lettre de l'AFRASE 2 (février 1985), http://www.afrase.org/wp-content/uploads/2014/11/1985Lettre-02.pdf (consulté le 15 mars 2016).

Lettre de l'AFRASE 3 (juin 1985), http://www.afrase.org/wp-content/uploads/2014/11/1985Lettre-03.pdf (consulté le 15 mars 2016).

BOURDEAUX, Pascal, « L'École Pratique des Hautes Études, les sciences religieuses et le Vietnam. À la croisée des situations coloniale et postcoloniales ", Revue d'Histoire des Sciences humaines 1/24 « Décolonisation et sciences humaines » (2011), p. 131-157.

CHARNASSE, Hélène (dir.), Aspects de la recherche musicologique au Centre national de la recherche scientifique, Paris, CNRS, 1984.

CLER, Jérôme et PICARD, François, « De la scène à l'estrade », Cahiers d'ethnomusicologie 20 (2007), p. 273-288, mis en ligne le 16 janvier 2012 : http://ethnomusicologie.revues.org/296 (consulté le 15 mars 2016).

COSTÈRE, Edmond, compte-rendu de «L La résonance dans les échelles musicales, Études réunies par Édith Weber, CNRS, Paris, 1963, ", Revue de musicologie 51/2 (1965), p. 241-243.

DUZAN, Brigitte « Je suis né un 4 juin : évocation cathartique des fantômes d'une époque par Liu Xinwu », 11 mai 2013, http://www.chinese-shortstories.com/Actualites_81.htm (consulté le 15 mars 2016).

O’REILLY, Patrick, compte-rendu de « Kelkel, Manfred. À la découverte de la Musique Polynésienne traditionnelle, Paris, Publications Orientalistes de France, 1981 ", Journal de la Société des océanistes 39/76 (1983), p. 129.

PICARD, François, « Tràn Van Khê, un parcours sans fautes » (entretien), Cahiers de musiques traditionnelles 2 (1990), p. 235-242.

PICARD, François, « La Revue musicale : Tràn Van Khê et le Vietnam », Cahiers de musiques traditionnelles 2 (1990), p. 282-283.

PICARD, François, « L'ethnomusicologie et Jacques Chailley », Musurgia XIX/1-3 « Jacques Chailley » (2012), p. 37-43.

REVEL, Nicole (dir.), Le Riz en Asie du Sud-Est : Atlas du vocabulaire de la plante, Paris, EHESS, Ateliers d'ASEMI, 1988.

TRAN, Van Khê, « Jacques Chailley et la promotion de l'ethnomusicologie », Musurgia XIX/ 1-3 « Jacques Chailley » (2012), p. 31-36.

\section{NOTES}

1. «1984-1987 : directeur du Séminaire sur “Les chants alternés en Asie du Sud Est” École des Hautes Études en Sciences Sociales ", http://www.philmultic.com/tran/french.html (consulté le 15 mars 2016).

2. Pour reprendre l'expression de Jérôme Cler. CLER, Jérôme et PICARD, François, « De la scène à l'estrade ", Cahiers d'ethnomusicologie 20 (2007), p. 273-288, mis en ligne le 16 janvier 2012 : http://ethnomusicologie.revues.org/296 (consulté le 15 mars 2016). 
3. Voir CHARNASSE, Hélène (éd.), Aspects de la recherche musicologique au Centre national de la recherche scientifique, Paris, CNRS, 1984.

4. TRAN, Van Khê, «Jacques Chailley et la promotion de l'ethnomusicologie », Musurgia XIX/1-3 "Jacques Chailley» (2012), p.31-36; PICARD, François, "L'ethnomusicologie et Jacques Chailley », Musurgia, XIX/1-3, « Jacques Chailley » (2012), p. 37-43.

5. DUZAN, Brigitte «Je suis né un 4 juin : évocation cathartique des fantômes d'une époque par Liu Xinwu », 11 mai 2013, http://www.chinese-shortstories.com/Actualites_81.htm (consulté le 15 mars 2016).

6. http://www.donbak.co.uk/artists/chemirani_djmachid.htm, http://www.villamedici.it/it/ event/musique-baroque-aux-portes-de-l-orient-ensemble-la-turchescha/?of=2\&t=musica\&p=all (consulté le 15 mars 2016).

7. «1959-1988 [je rectifie 1980 en 1988 en suivant la leçon de l'anglais]: Professeur, Directeur d'Études, puis Président du Centre d'Études de Musique Orientale (créé sous les auspices de l'Institut de Musicologie de Paris,) (Université de Paris-Sorbonne) », http://www.philmultic.com/ tran/french.html (consulté le 15 mars 2016).

8. « Alain Daniélou, conseilleur au Conseil international de la musique de l'UNESCO, directeur du Centre d'Etudes de Musique Orientale de Paris, Membre de l'École française d'Extrême-Orient ». Cité dans COSTÈRE, Edmond, compte-rendu de "La résonance dans les échelles musicales, Études réunies par Edith Weber, Paris, CNRS, 1963, », Revue de musicologie 51/2 (1965), p. 241.

9. Tran Van Khê, Président; Vadime Eliseef et Jean During, Vice-Présidents ; Denise JourdanHemmerdinger, Secrétaire générale ; Gilles Leothaud, Secrétaire général adjoint ; Tran Van Kim Ly, Trésorière, adjointe administrative ; direction des études : Alain Danielou, Tran Van Khê, Jean During. Source : Centre d'études de musique orientale, Bulletin 30 (1984).

10. O’REILLY, Patrick, compte-rendu de «Kelkel, Manfred. À la découverte de la Musique Polynésienne traditionnelle, Paris, Publications Orientalistes de France, 1981 », Journal de la Société des océanistes 39/76 (1983), p. 129.

11. Lettre de l'AFRASE 2 (février 1985), p.11, http://www.afrase.org/wp-content/uploads/ 2014/11/1985-Lettre-02.pdf (consulté le 15 mars 2016). Les prénoms et affiliations sont mentionnés dans la Lettre de l'AFRASE 3 (juin 1985), p. 3, http://www.afrase.org/wp-content/ uploads/2014/11/1985-Lettre-03.pdf (consulté le 15 mars 2016).

12. REVEL, Nicole (éd.), Le Riz en Asie du Sud-Est: Atlas du vocabulaire de la plante, Paris, EHESS, Ateliers d'ASEMI, 1988.

13. PICARD, François, "Tràn Van Khê, un parcours sans fautes » (entretien), Cahiers de musiques traditionnelles 2 (1990), p. 235-242 ; id., "La Revue musicale : Tràn Van Khê et le Vietnam », Cahiers de musiques traditionnelles 2 (1990), p. 282-283.

14. Le cours que Tran consacre aux chants alternés vient cinquante ans après la première thèse d'un Vietnamien, Nguyễn Văn Huyên, qui portait précisément sur ce sujet : « Sa thèse (Les chants alternés des garçons et des filles en Annam) et sa thèse complémentaire (l'habitat sur pilotis en Asie du Sud-Est) - notons au passage l'utilisation avant-gardiste du terme "Asie du Sud-Est" - sont publiées en 1934 à Paris chez Geuthner ». BOURDEAUX, Pascal, "L'École Pratique des Hautes Études, les sciences religieuses et le Vietnam. À la croisée des situations coloniale et postcoloniales", Revue d'Histoire des Sciences humaines $1 / 24$ "Décolonisation et sciences humaines » (2011), p. 131-157. 


\section{AUTEUR}

\section{FRANÇOIS PICARD}

François Picard est professeur d'ethnomusicologie à Sorbonne Université. Il a suivi des études de théâtre à Paris 8, puis opte pour les musiques Renaissance, puis chinoises au CEMO, où il devient disciple de Tran Van Khê. Il entreprend alors un doctorat de musicologie à Paris I (Iannis Xenakis), et se rend au conservatoire de musique de Shanghai. Après avoir travaillé comme directeur artistique en musiques traditionnelles et comme producteur pour France Musique et France Culture, il est Senior researcher à l'International Institute for Asian Studies (Leiden), puis soutient son HDR à l'EHESS sous la double tutelle de Pierre-Etienne Will et François-Bernard Mâche. Il a été responsable du DEA puis directeur de la mention Master, directeur de l'équipe Patrimoines et Langages Musicaux et responsable pour Paris-Sorbonne de la chaire Geste Acoustique Musique de Sorbonne Universités. Il a été l'organisateur principal de deux conférences internationales: Chime et Luoshen fu arts et humanités. Il joue de la flûte xiao et de l'orgue à bouche sheng au sein de l'ensemble XVIII-21, le baroque nomade et collabore avec des compositeurs contemporains (Tehericsen, Bernard, Lévy). Publications : La Musique chinoise, Paris, You-Feng (2003), Lexique des musiques d'Asie orientale, Paris, You-Feng (2006), L'incantion du patriarche Pu'an. Les avatars du syllabaire sanskrit dans la musique chinoise, Leuven, Peeters, « Mélanges chinois et bouddhiques » XXXI (2012). http://www.iremus.cnrs.fr/membrespermanents/francois-picard 